\title{
Developmental Programming and Polycystic Ovarian Syndrome
}

\section{Maria Rosa Maduro, PhD}

Developmental programming refers to the response by the developing mammalian organism to a specific challenge during prenatal or early postnatal development that permanently alters the physiology, metabolism or epigenome of an individual with subsequent consequences to this individual's health and increased risk of certain diseases later in life. It constitutes an important and rapidly englobing concept in Science, as more and more evidence on the impact of developmental programming on health and disease becomes evident through animal model, clinical and epidemiological studies. ${ }^{1}$

Examples of fetal or early postnatal exposures impacting different aspects of reproductive biology have naturally been published in Reproductive Sciences and the topic is once more revisited in the current issue of the journal through the work by Puttabyatappa et al. ${ }^{2-8}$

Given the similarities in the ovarian phenotype between women with polycystic ovarian syndrome (PCOS) and sheep prenatally treated with testosterone, and the fact that women with PCOS are known to have an increased activity of ovarian matrix metalloproteinases (MMP), Puttabyatappa and colleagues hypothesized that gestational excess testosterone may be on the basis of PCOS by altering ovarian expression of MMPs and its target regulators.

Using the sheep as a model, the authors used immunohistochemistry to determine the ovarian content of the MMP inhibitor TIMP and the target proteins laminin B (LAMB), collagen, tumor necrosis factor alpha (TNF) and connexin 43(GJA1) at fetal days 90, 140 and 21 months of age (adult).

Puttabyatappa and colleagues observed that prenatal testosterone exposure in excess lowered the GJA1 protein content in stroma and granulosa cells of primary follicles from fetal day 90 ovaries, while it decreased the stromal expression of MMP9, TIMP1 and LAMB in ovaries from fetal day 140 sheep. In addition, the authors noted that in the adult sheep, prenatal treatment with testosterone led to increased expression of MMP9 in theca cells of large preantral follicles and stroma, while TNF was elevated in granulosa cells of small and large preantral follicles and theca cells of large preantral and antral follicles. GJA1 expression, in turn, was in the adult sheep elevated in stroma, theca cells of large preantral follicles and granulosa cells of antral follicles. On the other hand, Puttabyatappa et al. observed that after excess testosterone fetal exposureTIMP1 expression deceased in stroma and theca cells of large preantral and antral follicles, while LAMB was reduced in stroma and small preantral follicles and collagen expression decreased in the stroma and the cells surrounding antral follicles.
Thus, altogether, the results presented by Puttabyatappa and colleagues support their hypothesis that gestational exposure to excess testosterone alters the ovarian MMPs' activity pathway in sheep, potentially leading to the development of the multifollicular morphology characteristic of PCOS. Moreover, this work further emphasizes the importance of developmental programming in reproductive system's pathologies, namely in PCOS, shedding light into a mechanism likely to be associated with the development of this disease that may be therapeutically counteracted to avoid it.

\section{References}

1. Sutton EF, Gilmore LA, Dunger DB, Heijmans BT, Hivert MF, Ling C, Martinez JA, Ozanne SE, Simmons RA, Szyf M, Waterland RA, Redman LM, Ravussin E. Developmental programming: state-of-thescience and future directions-Summary from a Pennington Biomedical symposium. Obesity (Silver Spring). 2016;24(5):1018-1026.

2. Benesh EC, Gill J, Lamb LE, Moley KH. Maternal obesity, cage density, and age contribute to prostate hyperplasia in mice. Reprod Sci. 2016;23(2):176-185.

3. Khot VV, Chavan-Gautam P, Mehendale S, Joshi SR.Variable methylation potential in preterm placenta: implication for epigenetic programming of the offspring. Reprod Sci. 2017;24(6):891-901.

4. Hewlett M, Chow E, Aschengrau A, Mahalingaiah S. Prenatal exposure to endocrine disruptors: a developmental etiology for polycystic ovary syndrome. Reprod Sci. 2017;24(1):19-27.

5. Chen PY, Chu A, Liao W, Rubbi L, Janzen C, Hsu F, Thamotharan S, Ganguly A, Lam L, Montoya D, Pellegrini M, Devaskar SU. Prenatal growth patterns and birthweight are associated with differential DNA methylation and gene expression of cardiometabolic risk genes in human placentas: a discovery-based approach. Reprod Sci. 2018;25(4):523-539.

6. Gao Y, Chen F, Kong Q, Ning S, Yuan H, Lian H, Luo M, Tan J. Stresses on female mice impair oocyte developmental potential: effects of stress severity and duration on oocytes at the growing follicle stage Reprod Sci. 2016;23(9):1148-1157.

7. Zou T, He D, Yu B, Yu J, Mao X, Zheng P, He J, Huang Z, Chen D. Moderate maternal energy restriction during gestation in pigs attenuates fetal skeletal muscle development through changing myogenic gene expression and myofiber characteristics. Reprod Sci. 2017;24(1):156-167.

8. Puttabyatappa M, Irwin A, Martin JD, Mesquitta M, Veiga-Lopez A, Padmanabhan V. Developmental programming: gestational exposure to excess testosterone alters expression of ovarian matrix metalloproteases and their target proteins. Reprod Sci. 2018;25(6): 882-892. 